\title{
A Cross-sectional Survey of Internal Medicine Resident Knowledge, Attitudes, Behaviors, and Experiences Regarding Pre-Exposure Prophylaxis for HIV Infection
}

\author{
Christopher Terndrup, $M D^{7}$, Carl G. Streed, Jr., MD, MPH², Perry Tiberio, MD, PhD ${ }^{3}$, \\ Marissa Black, MD, MPH', John Davis, MD, PhD ${ }^{5}$, Ariella Apfel, MPH', Oni J. Blackstock, MD, MHS', \\ E. Jennifer Edelman, $M D, M H S^{8}$, and Gail Berkenblit, $M D, P h D^{6}$
}

\begin{abstract}
'Department of Medicine, Division of General Internal Medicine and Geriatrics, Oregon Health and Sciences University, Portland, OR, USA; ${ }^{2}$ Section of General Internal Medicine, Boston Medical Center, Boston University School of Medicine, Boston, MA, USA; ${ }^{3}$ Department of Medicine, Columbia University Medical Center, New York, NY, USA; ${ }^{4}$ Division of Gerontology and Geriatric Medicine, University of Washington, Seattle, WA, USA; ${ }^{5}$ Division of Infectious Disease, Department of Medicine, University of California at San Francisco, San Francisco, CA, USA; ${ }^{6}$ Division of General Internal Medicine, Department of Medicine, Johns Hopkins University School of Medicine, Baltimore, MD, USA; ${ }^{7}$ Montefiore Medical Center/Albert Einstein College of Medicine, Bronx, NY, USA; ${ }^{8}$ Division of General Internal Medicine, Yale School of Medicine, New Haven, CT, USA.
\end{abstract}

BACKGROUND: Pre-exposure prophylaxis (PrEP) for HIV is effective, yet many providers continue to lack knowledge and comfort in providing this intervention. It remains unclear whether internal medicine (IM) residents receive appropriate training in PrEP care and if this affects their future practices.

OBJECTIVE: We sought to evaluate the relationship between current IM residents' prior PrEP training and knowledge, comfort, and practice regarding the provision of PrEP.

DESIGN AND PARTICIPANTS: We created an online survey to assess IM residents' knowledge, attitudes, and behaviors related to PrEP. The survey was distributed among five IM programs across the USA.

KEY RESULTS: We had a 35\% response rate. Of 229 respondents, 96\% $(n=220)$ had heard of PrEP but only $25 \%(n=51)$ had received prior training and $11 \%(n=$ 24) had prescribed PrEP. Compared with those without, those with prior training reported good to excellent knowledge scores regarding PrEP (80\% versus 33\%, $p<0.001)$, more frequent prescribing (28\% versus $7 \%$, $p=0.001$ ), and higher comfort levels with evaluating risk for HIV, educating patients, and monitoring aspects of PrEP (75\% versus $26 \%, 56 \%$ versus $16 \%$, and $47 \%$ versus $8 \%$, respectively; all $p$ values $<0.0001$ ). While only $25 \%(n=51)$ had received prior training, $75 \%(n=103)$ of respondents reported that training all providers at their continuity clinic sites would improve implementation.

CONCLUSIONS: We found that prior training was associated with higher levels of self-reported PrEP knowledge, comfort, and prescribing behaviors. Given the significant need for PrEP, IM residents should be trained to achieve adequate knowledge and comfort levels to prescribe it. This study demonstrates that providing appropriate PrEP training for IM residents may lead to an increase in the pool of graduating IM residents prescribing PrEP.

KEY WORDS: HIV pre-exposure prophylaxis; resident education.

Published online April 24, 2019
$\mathrm{J}$ Gen Intern Med 34(7):1258-78

DOI: $10.1007 / \mathrm{s} 11606-019-04947-2$

(c) Society of General Internal Medicine 2019

\section{INTRODUCTION}

Despite the proven effectiveness of pre-exposure prophylaxis (PrEP) against the acquisition of the human immunodeficiency virus (HIV) among high-risk individuals, ${ }^{1,2}$ provider adoption remains low. Emtricitabine/tenofovir disoproxil fumarate (FTC/TDF, trade name Truvada ${ }^{\circledR}$, Gilead Sciences Inc.), the only FDA-approved form of HIV PrEP, has been shown in multiple studies to reduce the risk of HIV infection in adults with high-risk sexual activity and persons with injection drug use. ${ }^{3}$ The Centers for Disease Control and Prevention (CDC) guidelines have been in place since $2014,{ }^{4}$ but prior studies have shown significant barriers to prescribing PrEP, from lack of provider knowledge and comfort to concerns for cost, side effects, and increased risk in sexual behavior. ${ }^{5-8}$

Studies suggest that addressing knowledge and skills gaps in these areas is a key to increasing provision of PrEP. A qualitative survey of providers revealed significant knowledge gaps in identifying target populations, prescribing, and monitoring of PrEP and a lack of consensus among protocols. ${ }^{9}$ Canadian clinicians face similar barriers, with over $75 \%$ reporting that information has not been adequately disseminated to physicians and only $12 \%$ reporting having prescribed PrEP. ${ }^{10}$ Increased knowledge of PrEP is associated with higher rates of past prescriptions and future intent to prescribe, regardless of provider type. ${ }^{7}$ Among general internists, both self-reported knowledge and experience with HIV care have been shown to correlate with higher rates of PrEP prescribing. ${ }^{11}$ At-risk populations also face knowledge barriers and importantly, having a primary care physician who was aware of their risk status has been shown to be associated with increased patient awareness of PrEP. ${ }^{12-14}$ 
While barriers exist across the care spectrum, medical educators are well-suited to address training and behavior concerns during residency in order to help close this gap in care. While didactics like continuing medical education (CME) can improve knowledge scores, they often fail to translate into practice change. ${ }^{15}$ On the other hand, factors that have been shown to improve implementation of guidelines into practice (e.g., individual face-to-face teaching, a supportive environment, and influence of well-respected physician champions) are common components of residency training. ${ }^{16}$ Training during residency affords the opportunity for additional reinforcement including delineation of expected practice patterns, directed audit, and feedback and active intervention within the clinical setting. ${ }^{17}$ Clinician educators are role models in encouraging resident adoption of guidelines including HIV testing ${ }^{18}$ and resident practice has been shown to reflect preceptor prescribing practices. ${ }^{19,} 20$

A 2015 survey $^{11}$ of members of the Society of General Internal Medicine (SGIM) found that of the resident respondents, $22 \%$ reported PrEP adoption, significantly fewer than the $38 \%$ of attending physicians surveyed. However, this survey had fewer than 50 resident respondents, and no other studies have assessed resident populations. To establish baseline characteristics of this population and understand the role of training for PrEP, we sought to understand the current self-reported knowledge, attitudes, behaviors, and experiences of IM residents in using PrEP. Based on prior research, we hypothesize that adequate PrEP training during IM residency would increase the number of current and future PrEP prescribers.

\section{METHODS}

From April through June of 2016, we conducted a survey of self-reported PrEP knowledge, attitudes, behavior, and experience among IM residents at five academic medical centers: Johns Hopkins Hospital, Johns Hopkins Bayview Medical Center, Yale School of Medicine, University of Washington, and Ohio State University. The survey was adapted from a prior PrEP survey of Society of General Internal Medicine (SGIM) members ${ }^{11}$ which assessed self-reported knowledge of and prior training regarding PrEP, its efficacy and side effects, and practice characteristics and adoption of PrEP prescribing. Additional questions were added to investigate issues specific to training and resident experience (i.e., how faculty supervision may have affected prescribing behavior, preferred implementation strategy in resident clinics, and barriers to prescribing PrEP). After confirming residency status, the survey provided a brief description of PrEP and a formal definition but also noting the common terminology of Truvada as equivalent to PrEP. Questions regarding self-reported basic knowledge of PrEP and its side effects followed, including specific questions regarding training during residency. Finally, we assessed resident comfort levels in prescribing PrEP. We finished by asking about their prior prescribing habits (see Appendix 1 for full survey). The 50-question survey was administered in English and included questions regarding demographics and practice characteristics. We piloted our adaptation of the survey with four resident colleagues in training across the USA with experience in HIV PrEP and survey design to confirm acceptability of survey length, content, and readability of questions.

All residents in five residency programs received recruitment emails for the online survey on Qualtrics via program listservs sent by study coordinators at each site. Two follow-up emails were sent over the 2-month period as reminders to complete the survey. The recruitment email included a brief introduction to the survey, along with a disclosure and the notation of a raffle drawing for a \$20 Amazon gift card. Institutional review board exempt status from Johns Hopkins University School of Medicine was granted for this project prior to the release of the survey to the subjects. Surveys were excluded in the case of preliminary training status (neurology interns, $n=2$ ). Not all respondents completed all survey items and we have shown the number of responses per item.

In terms of statistical analysis, we ran a chi-square analysis for our categorical data using Statistical Analysis System (SAS) 9.4 to see if there were any significant associations between survey items and whether residents had received prior training on PrEP. Fisher's exact tests were performed instead of chi-square analyses when cell counts were too low for chi-square tests to be reliable. Due to small sample size in certain categories, we were unable to perform multivariate analyses.

\section{RESULTS}

We obtained a response rate of $35 \%$ based on residency program sizes and the number of submitted surveys $(n=$ 229) (Table 1). The response rates from the five sites ranged from 13 to $47 \%$. Our respondents did not differ significantly by age, race, or ethnicity from nationwide demographics for internal medicine residents, but did have a higher proportion of women $(52 \%$ versus $43 \%, p=$ $0.02){ }^{21}$ The average age of respondents was 30 years old (age range 23-41) and the sample was relatively even among self-identified men and women and among training years, with a slightly higher representation of PGY-1 level residents. A majority of respondents self-identified as White or Asian. The vast majority (95\%) reported a heterosexual orientation. Beyond a slight trend among nonheterosexual respondents, there were no statistically significant demographic differences among those that received training and those that had not. 
Table 1 Respondent Characteristics by Self-reported Training in PrEP

\begin{tabular}{|c|c|c|c|c|c|}
\hline & Total & Prior training on PrEP & $\begin{array}{l}\text { No prior training } \\
\text { on PrEP }\end{array}$ & $\begin{array}{l}\text { Chi-square statistic } \\
\text { *Fisher's exact test }\end{array}$ & $p$ value \\
\hline \multicolumn{4}{|l|}{ Race/ethnicity } & * & 0.27 \\
\hline White & $129(63 \%)$ & $38(75 \%)$ & $91(60 \%)$ & & \\
\hline Asian & $51(25 \%)$ & $8(16 \%)$ & $43(28 \%)$ & & \\
\hline African-American & $9(4 \%)$ & $2(4 \%)$ & $7(5 \%)$ & & \\
\hline Other & $15(7 \%)$ & $3(6 \%)$ & $12(8 \%)$ & & \\
\hline \multicolumn{4}{|l|}{ Hispanic } & \multirow[t]{4}{*}{$*$} & 1.00 \\
\hline Yes & $10(5 \%)$ & $2(4 \%)$ & $8(5 \%)$ & & \\
\hline No & $191(94 \%)$ & $48(94 \%)$ & $143(94 \%)$ & & \\
\hline Prefer not to say & $3(1 \%)$ & $1(2 \%)$ & $2(1 \%)$ & & \\
\hline \multicolumn{4}{|l|}{ Gender } & \multirow[t]{3}{*}{2.2} & \multirow[t]{3}{*}{0.13} \\
\hline Man & $98(48 \%)$ & $20(39 \%)$ & $78(51 \%)$ & & \\
\hline Woman & $105(52 \%)$ & $31(60 \%)$ & $74(49 \%)$ & & \\
\hline \multicolumn{4}{|l|}{ Sexual orientation } & \multirow[t]{3}{*}{$*$} & \multirow[t]{3}{*}{0.13} \\
\hline Heterosexual & $190(95 \%)$ & $46(90 \%)$ & $144(97 \%)$ & & \\
\hline Other & $10(5 \%)$ & $5(10 \%)$ & $5(3 \%)$ & & \\
\hline \multicolumn{4}{|l|}{ PGY level } & \multirow[t]{4}{*}{3.0} & \multirow[t]{4}{*}{0.22} \\
\hline PGY1 & $78(38 \%)$ & $15(29 \%)$ & $63(41 \%)$ & & \\
\hline PGY2 & $67(33 \%)$ & $17(33 \%)$ & $50(34 \%)$ & & \\
\hline PGY3/4 & $59(29 \%)$ & $19(37 \%)$ & $40(26 \%)$ & & \\
\hline \multicolumn{4}{|l|}{ Location } & \multirow[t]{5}{*}{5.8} & \multirow[t]{5}{*}{0.12} \\
\hline Connecticut & $56(28 \%)$ & $15(30 \%)$ & $41(27 \%)$ & & \\
\hline Maryland & $92(46 \%)$ & $18(36 \%)$ & $74(49 \%)$ & & \\
\hline Ohio & $17(9 \%)$ & $3(6 \%)$ & $14(9 \%)$ & & \\
\hline Washington & $36(18 \%)$ & $14(28 \%)$ & $22(15 \%)$ & & \\
\hline
\end{tabular}

*Fisher's exact testing was used

Of those surveyed, 96\% had heard of PrEP. Threequarters of the sample received no prior training on PrEP with no significant differences among the programs surveyed. Respondents who had received prior training reported lecture, discussion, or practice-based protocol review as the source of their prior knowledge of PrEP. Nevertheless, nearly $90 \%$ of all respondents regardless of prior training believed they needed more education prior to feeling comfortable prescribing PrEP. More than half rated their knowledge of the medication (55\%) and its side effects $(76 \%)$ as only poor or fair. While only $11 \%$ had prescribed PrEP during their residency, $85 \%$ said that they would. A majority of respondents $(84 \%)$ believed their attending would be supportive of PrEP prescribing by a resident.

Those who had received prior training in PrEP differed significantly in self-reported knowledge, attitudes, and practice from those who had not received training (Table 2). Residents who had received training were much more likely to report better knowledge of PrEP and its side effects and were also more likely to endorse its efficacy. Residents who rated their knowledge more highly reported a greater likelihood of prescribing PrEP in the future (Fig. 1).

To further understand the impact of training on willingness to prescribe PrEP, we evaluated resident comfort level with identifying target populations based on CDC recommendations, providing patient education and monitoring therapy (Fig. 2). Residents with prior training were much more comfortable evaluating eligibility for PrEP, educating patients on its use, and monitoring for side effects, toxicity, and requirements for screening for sexually transmitted infections (STIs). Lastly, we assessed the impact of training on resident willingness to prescribe PrEP among different risk groups (Fig. 3 in the Appendix 2). Training was associated with increased willingness to prescribe PrEP for MSM and for HIV negative females coupled with HIVpositive male partners. No differences were seen in willingness to prescribe for other serodiscordant couples, where willingness was high in both groups. All residents, those who had received training as well as those who had not, reported comparative reluctance to prescribe PrEP to persons with active injection drug use.

Residents noted several barriers to prescribing PrEP during their training (Table 3). The most common barriers were ranked on a Likert scale. Lack of education, lack of clinic protocols, and lack of preceptor support were larger barriers for those who had not done prior training. In light of these barriers, when asked about the most feasible approach to PrEP implementation at their residency clinics, $70 \%$ of the all respondents believed that all providers should receive training about PrEP, while only $22 \%$ and $6 \%$ believed it best to have an onsite PrEP specialist or referral to an outside provider, respectively.

\section{DISCUSSION}

This study provides new information regarding lack of training among IM residents for PrEP. Our findings expand upon a prior survey of SGIM members, ${ }^{11}$ confirming low numbers of "adopters" among residents while identifying specific areas for improvement, with recommendations from the residents themselves. Although the majority of IM residents had heard of PrEP, few had received PrEP training and even fewer had prescribed PrEP. The majority reported a perceived need for more education before 
Table 2 Knowledge, Attitudes, and Practices Related to PrEP by Self-reported Training in PrEP

\begin{tabular}{|c|c|c|c|c|c|}
\hline & Total & Prior training on PrEP & $\begin{array}{l}\text { No prior training } \\
\text { on PrEP }\end{array}$ & $\begin{array}{l}\text { Chi-square statistic } \\
\text { *Fisher's exact test }^{-}\end{array}$ & $p$ value \\
\hline \multicolumn{6}{|l|}{ Knowledge } \\
\hline Knowledge (general) & & & & 52 & $<0.001$ \\
\hline Poor/fair & $121(55 \%)$ & $11(20 \%)$ & $110(67 \%)$ & & \\
\hline Good & $57(26 \%)$ & $17(31 \%)$ & $40(24 \%)$ & & \\
\hline Very good/excellent & $42(19 \%)$ & $27(49 \%)$ & $15(9 \%)$ & & \\
\hline Knowledge of side effects & $167(76 \%)$ & $24(44 \%)$ & $143(87 \%)$ & 53 & $<0.001$ \\
\hline Poor/fair & $31(14 \%)$ & $13(24 \%)$ & $18(11 \%)$ & & \\
\hline Good & $22(10 \%)$ & $18(33 \%)$ & $4(2 \%)$ & & \\
\hline \multicolumn{6}{|l|}{ Very good/excellent } \\
\hline \multicolumn{6}{|l|}{ Attitudes } \\
\hline Efficacy & & & & $*$ & 0.53 \\
\hline Disagree & $7(3 \%)$ & $0(0 \%)$ & $7(4 \%)$ & & \\
\hline Agree & $205(97 \%)$ & $53(100 \%)$ & $152(96 \%)$ & & \\
\hline Safety & & & & $*$ & 0.73 \\
\hline Disagree & $14(7 \%)$ & $2(4 \%)$ & $12(8 \%)$ & & \\
\hline Agree & $198(93 \%)$ & $51(96 \%)$ & $147(92 \%)$ & & \\
\hline Increased risk behaviors & $163(79 \%)$ & $42(79 \%)$ & $121(79 \%)$ & 0.22 & 0.64 \\
\hline Disagree & $44(21 \%)$ & $11(21 \%)$ & $33(21 \%)$ & & \\
\hline \multirow{2}{*}{\multicolumn{6}{|c|}{ Practice }} \\
\hline & & & & & \\
\hline Likely to prescribe & & & & 16 & $<0.001$ \\
\hline Disagree & $153(72 \%)$ & $27(51 \%)$ & $126(79 \%)$ & & \\
\hline Agree & $59(28 \%)$ & $26(49 \%)$ & $33(21 \%)$ & & \\
\hline Prescribed before & & & & 18 & $<0.001$ \\
\hline No, and would not & $7(3 \%)$ & $0(0 \%)$ & $7(4 \%)$ & & \\
\hline No, but would & $181(85 \%)$ & $39(74 \%)$ & $142(89 \%)$ & & \\
\hline Yes & $24(11 \%)$ & $14(26 \%)$ & $10(6 \%)$ & & \\
\hline Initiated conversation & & & & 19 & $<0.001$ \\
\hline No & $156(74 \%)$ & $27(51 \%)$ & $129(81 \%)$ & & \\
\hline Yes & $56(26 \%)$ & $26(49 \%)$ & $30(19 \%)$ & & \\
\hline Referred for PrEP & & & & 0.32 & 0.58 \\
\hline No & $181(85 \%)$ & $44(83 \%)$ & $137(86 \%)$ & & \\
\hline Yes & $31(15 \%)$ & $9(17 \%)$ & $22(14 \%)$ & & \\
\hline
\end{tabular}

*Fisher's exact testing was used

feeling comfortable prescribing PrEP. In contrast to postgraduate physicians, ${ }^{22}$ most felt that all providers should receive specific training for PrEP, while a minority felt that a PrEP specialist or referral base would be more prudent. Our findings are the first to our knowledge looking specifically at IM residents.
Guidelines can influence physician behavior and promote adoption of new practices such as $\mathrm{PrEP}^{23}$; however, effectively enacting guidelines requires translation into practice. Studies have shown that while dissemination of guidelines is important, their implementation is best achieved by multifaceted approaches that incorporate

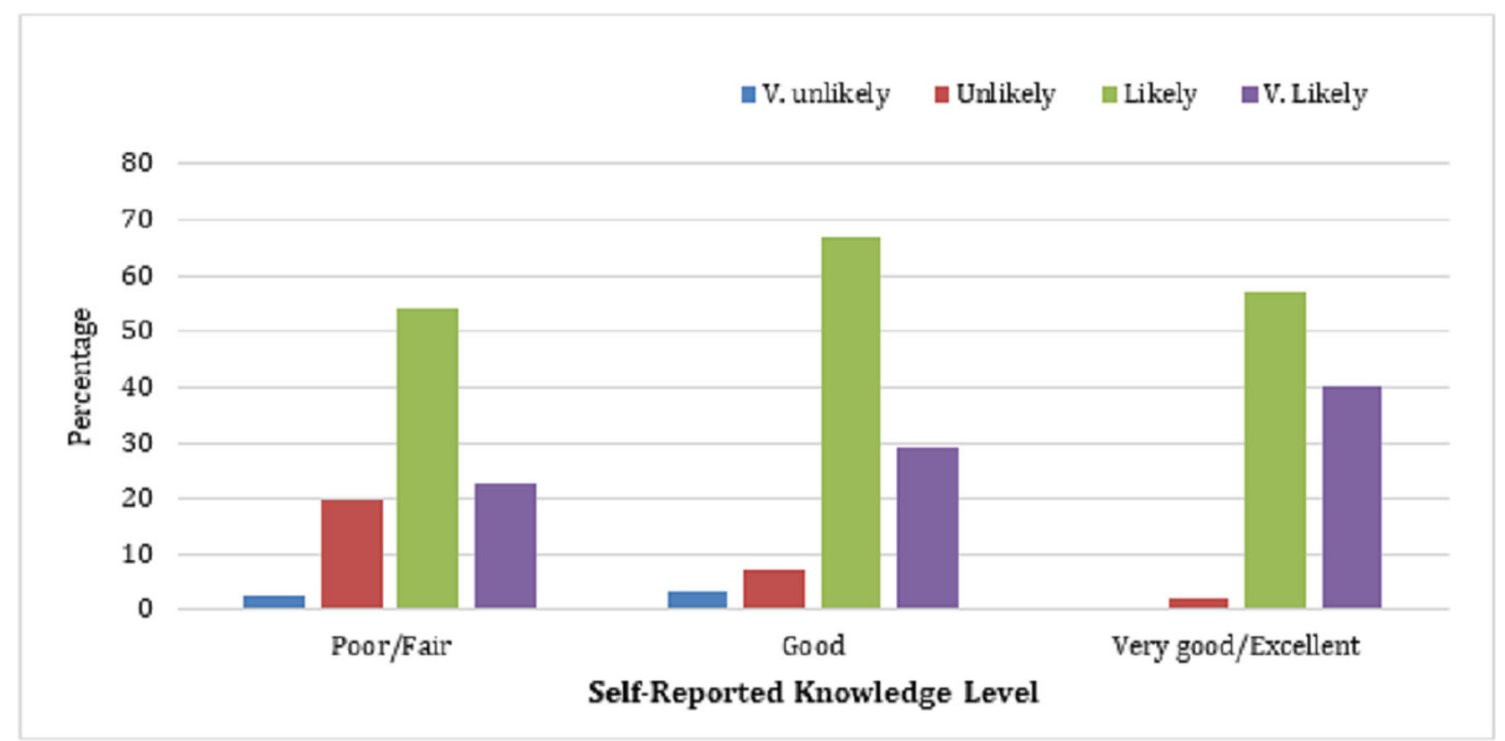

Figure 1 Likelihood of prescribing PrEP by self-reported knowledge. Percent of residents within each self-reported knowledge level reporting likelihood of prescribing PrEP in the next 6 months. 

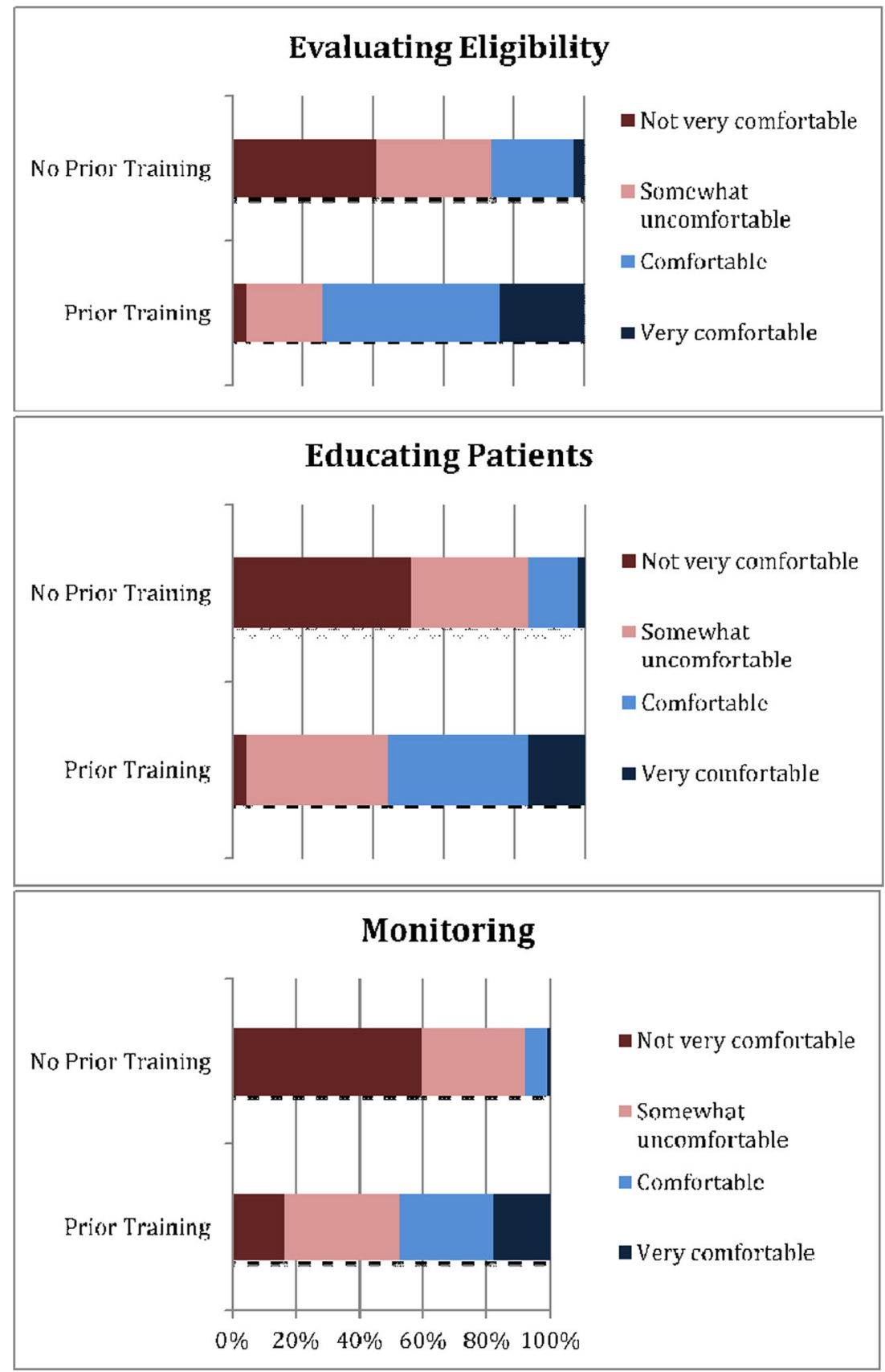

Figure 2 Comfort levels with evaluation, education, and monitoring for PrEP: comparison of residents who report receiving training versus those who did not. All $p$ values less than 0.01 .

active learning and feedback. ${ }^{24}$ Residency training combines aspects of didactic training as well as role modeling, audit, and feedback. Additionally, studies show that young physicians may be more open to adoption and change. ${ }^{25}$ Thus, as suggested by this study, starting at the graduate medical education (GME) level may prove very important in educating a healthcare workforce comfortable and trained in prescribing PrEP.

Our survey found that training is associated with a significant improvement in resident self-reported knowledge, attitudes, and intent to prescribe. Currently, the majority of resident PrEP education is delivered through lecture, discussion, or review of a protocol. In addition, a high percentage of residents reported clinical mentors that were supportive - thus, residency has a unique infrastructure to teach and translate guidelines into practice. However, less than a third of residents, even among PGY-3s, reported prior training. This suggests that training is not being delivered uniformly. While this study did not discern the most effective way to train residents, be it through work- 
Table 3 Perceived Barriers to PrEP Among All Respondents, the Likert Scale 1-4 (1 = Not At All Likely to be a Barrier; $4=$ Extremely Likely to be a Barrier)

\begin{tabular}{ll}
\hline \hline Barrier & $\begin{array}{l}\text { Average } \\
\text { ranking }\end{array}$ \\
\hline Lack of provider training/education regarding PrEP* & 3.36 \\
Lack of clinic guidelines/protocols for PrEP* & 2.75 \\
Lack of insurance coverage/ out-of-pocket costs & 2.59 \\
Time and staffing constraints related to counseling & 2.39 \\
Clinical and lab monitoring requirements & 2.24 \\
Lack of preceptor support regarding PrEP* & 2.10 \\
\hline
\end{tabular}

“*” indicates a larger barrier for those without prep training

shops, seminars, or OSCEs (objective structured clinical examinations), ${ }^{23,}{ }^{26}$ determining efficacy and then developing a format which can be widely disseminated within and across programs will be key.

Self-reported knowledge of PrEP was highest among those who reported training. As in other studies, those with higher knowledge were more likely to report intention to prescribe PrEP. ${ }^{23}$ Reported confidence in educating patients was also higher among residents who received prior training. Patient education regarding PrEP has been identified in other studies as another means of increasing uptake of HIV prevention. ${ }^{12-14}$ Self-reported knowledge of side effects and comfort with monitoring parameters was the lowest scoring areas for all residents. Providing further training and tools like standard protocols may be beneficial to address these learning needs. ${ }^{27}$

Because provision of PrEP relies on initial risk assessment, comfort with discussion of high-risk behaviors is essential. $^{26}$ Those who received prior HIV PrEP training reported greater comfort with evaluating patients for PrEP. In practice, residents with prior training reported initiating a conversation about PrEP more than those without. However, similar to post-graduate physicians, ${ }^{28}$ over $25 \%$ were unwilling to prescribe PrEP in scenarios with active injection drug use. Thus, further training and efforts to reduce difference in attitudes towards those in this risk category may need to be explored and addressed during residency training.

Overall, barriers in resident practice were similar to those reported for practicing physicians, including knowledge, lack of established protocols, and concern for costs and insurance coverage. ${ }^{7,}{ }^{11}$ While the majority of all residents believe PrEP to be safe and efficacious, those with prior training were less likely to be concerned about increased high-risk behaviors. ${ }^{29}$ Importantly, lack of preceptor support was seen as a barrier, especially for those without prior training. This suggests that physician champions within the practice may facilitate increased PrEP prescribing, as seen in other studies. ${ }^{27,} 30$
Our study was subject to certain limitations. First, we surveyed five internal medicine programs; our results may not be generalizable across graduate medical education (GME), including community programs and rural locations which may have less exposure to PrEP programs, as well as other specialties who may prescribe PrEP (including Pediatrics, Obstetrics/Gynecology, and Family Medicine). Community and rural programs may be even more impactful on PrEP uptake and the HIV epidemic based on known disparities. ${ }^{31}$ Other knowledge gaps have been found when discussing family planning and fertility issues surrounding PrEP, and this may impact other GME curricula. ${ }^{32-34} \mathrm{We}$ did not survey other health professions training programs such as NP or PA programs that may prescribe PrEP. Second, we elected a convenience sample of residents on listservs. We received only a $35 \%$ response rate, which while similar to reported response rates for online surveys, ${ }^{35,}{ }^{36}$ increases the potential for bias. The results may also be skewed by response bias; residents with particular interest in the subject matter may be more likely to fill out the survey and have prior knowledge. However, removing this bias would only further highlight deficiencies in training outlined in our findings. While our study focused on provider-related barrier to PrEP, we did not assess knowledge or awareness of patient barriers such as insurance coverage or cost of laboratory requirements, and these have been found to have a profound impact on PrEP uptake. ${ }^{31}$ Finally, residents were asked to self-report their training, knowledge, and behaviors and PrEP assessment and prescribing were not directly evaluated.

Since the advent of PrEP and reports of increased uptake among both patients and providers, we have seen decreased incidence of HIV. ${ }^{4}$ As PrEP is an effective and underutilized tool in reducing the burden of HIV, residency programs should be preparing their residents to care for patients in need of PrEP. With an expanding role among primary care providers in prescribing $\operatorname{PrEP},{ }^{7}$ future internists represent a significant foundation for access to PrEP. Further studies are needed to find the optimal methods to teach trainees about PrEP to improve access to HIV prevention and reduce the incidence of HIV.

Acknowledgements: The authors would like to thank Amanda Bertram for her help with organizing the survey design and administration.

Corresponding Author: Christopher Terndrup, MD; Department of Medicine, Division of General Internal Medicine and Geriatrics Oregon Health and Sciences University, Portland, OR, USA (e-mail: terndrup@ohsu.edu).

Compliance with Ethical Standards:

Conflict of Interest: Marissa Black has stock in Gilead gifted by family in 2014, and all remaining authors declare that they do not have a conflict of interest. 


\section{APPENDIX 1}

Default Question Block

\section{Resident PrEP Survey}

Christopher Terndrup M.D., Carl G Streed Jr M.D., Gail Berkenblit M.D., Ph.D.

The purpose of this research study is to learn about internal medicine residents' training experience and knowledge, attitudes, and behaviors regarding pre-exposure prophylaxis ("PrEP") for HIV infection. This anonymous survey should take approximately 10 to 15 minutes to complete.

To thank you for your time, all participants will be entered into a raffle drawing for a $\$ 20$ Amazon gift card. There be 50 gfit cards total raffled in this manner.

By clicking the forward arrows at the bottom of this screen, you are agreeing to take part in this research study and understand that your responses will be used for research purposes. This research study is being administered through the Johns Hopkins University School of Medicine and has received IRB

(exemption/approval).

Your participation is completely voluntary and you do not have to participate if you do not want to; also you may skip questions you do not feel comfortable answering.

Browsers at work may have different security settings. If you experience any difficulties being directed to the survey or during the survey, try to open the link to the survey in a different browser or using a non-work computer or network. We also recommend that you complete this survey on a desktop computer or laptop. 
Please contact either Dr. Christopher Terndrup (cterndr1@jhmi.edu) or Dr. Carl G Streed Jr. (cstreed1@jhmi.edu) or Dr. Gail Berkenblit (gberken@jhmi.edu) with any questions.

Thank you for your time.

Are you currently completing a residency training program?

\section{Yes}

No

Based on your response, you are not eligible for this study. Thank you for your time.

PrEP stands for "Pre-Exposure Prophylaxis", the use of any medicine to prevent a disease before exposure to that disease. For the purposes of this survey, the term refers to the use of an oral antiretroviral medication taken on a daily basis by people at high risk of exposure to HIV to prevent HIV infection. Truvada, a combination of two antiretroviral medications tenofovir and emtricitabine, was approved for PrEP in 2012 by the FDA and recommended in 2014 by the CDC.

Before this survey, had you heard of PrEP?

$$
\begin{aligned}
& \text { Yes } \\
& \text { No }
\end{aligned}
$$

How would you rate your knowledge of PrEP?

Excellent

Very Good

Good

Fair

Poor 
How would you rate your knowledge of potential side effects associated with PrEP?

Excellent
Very Good
Good
Fair
Poor

The following set of questions asks about your training regarding PrEP:

Have you received specific training or education on PrEP?

$$
\text { Yes }
$$

No

What kind of training?

$$
\begin{gathered}
\text { Lecture } \\
\text { Discussion } \\
\text { Online Training Module } \\
\text { Protocol/Guidelines in practice setting }
\end{gathered}
$$

Other

Please answer the following regarding your comfort

$$
\begin{aligned}
& 1=\text { Not very } \\
& \text { comfortable }
\end{aligned}
$$

2

3

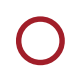

O for PrEP

Educating patients about PrEP
O

O
$4=$ Very comfortable 
Monitoring for adherence, toxicity, and STIs in patients on PrEP
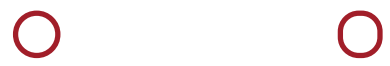

0

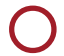

Would your attending/preceptor support you if you were to suggest PrEP for a patient?
Very Unlikely
Unlikely
Likely
Very Likely

Do you need more training before feeling comfortable prescribing PrEP?

Yes

No

For the following statement, please select all that apply:

There is good evidence that PrEP helps prevent HIV infection in:

Men who have sex with men

Heterosexual individuals

Sero-discordant couples (in which one individual is HIV positive and the other is HIV negative)

Intravenous drug users

The following set of questions asks about your opinions based on what you know about PrEP. It's OK if you're not very familiar with PrEP - just answer to the best of your ability. $(1=$ not at all; 4 = very)

$$
\begin{aligned}
& 1=\text { Not at } \\
& \text { all }
\end{aligned}
$$

How effective do you think PrEP is in preventing acquisition of HIV among people who take it every day as prescribed? 
consistently and correctly, how important

0

0

0

is it to offer PrEP in addition to condoms?

Based on your understanding of PrEP side effects, how safe is PrEP?

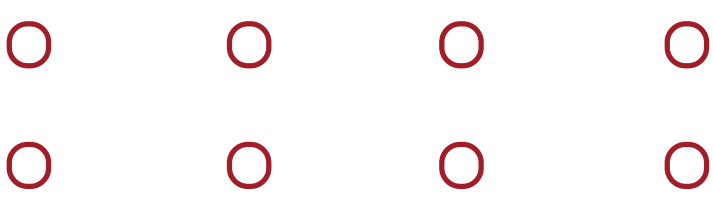

How likely are you to
the next 6 months?

If you identified a patient at high risk for HIV acquisition, how comfortable are you with prescribing PrEP?

How likely do you think the patient would be to increase his/her sexual risk-taking practices (e.g., decrease condom use) as a result of being on PrEP?

How likely do you think the patient would be to decrease his/her sexual risk-taking practices (e.g., increase condom use) as a result of being on PrEP?

$0 \quad 0$

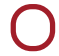

Have you ever been asked about PrEP by a patient?

Yes

No

Have you ever initiated a conversation about PrEP with a patient?

Yes

No

Have you ever prescribed PrEP to a patient?

\author{
Yes \\ No, but would \\ No, and would not
}

If you would not prescribe PrEP for at-risk patients, what are some concerns/reasons for not prescribing? (select all that apply) 
PrEP is not relevant to my practice

Patients are not at risk

Patients may engage in riskier behavior while on PrEP

Likelihood of low adherence to PrEP

Likelihood of HIV resistance developing

Cost and Payment Issues

Safety concerns regarding side-effects/toxicity

Concern about efficacy of real-world use of PrEP

Insufficient evidence

Other

Have you ever referred a patient for PrEP (e.g. to a PrEP provider or ID/HIV clinic)?

\section{Yes}

No

Rate the degree to which each of the following is a potential barrier to prescribing PrEP at your primary clinic: ( $1=$ not at all likely to be a barrier; $4=$ extremely likely to be a barrier)

Lack of provider training/education regarding PrEP

Lack of preceptor support regarding PrEP

O

$\mathrm{O}$

O prescribing/monitoring PrEP
O

$\mathrm{O}$

$\mathrm{O}$

$\mathrm{O}$

$\mathrm{O}$

$\mathrm{O}$

O

$\mathrm{O}$

$\mathrm{O}$ (also medication knowledge/counseling re: Truvada, adverse effects, etc.)

Lack of insurance coverage and out-ofpocket patient costs for PrEP and related

Clinical and lab monitoring requirements and STI screening every 3 months; checking renal function every 6 months) Staffing/time constraints related to risk
reduction and PrEP adherence counseling 
care (e.g., lab work)

Rate the degree to which each of the following would facilitate your prescribing PrEP at your primary clinic: (1= not at all likely to facilitate; 4 = extremely likely to facilitate)

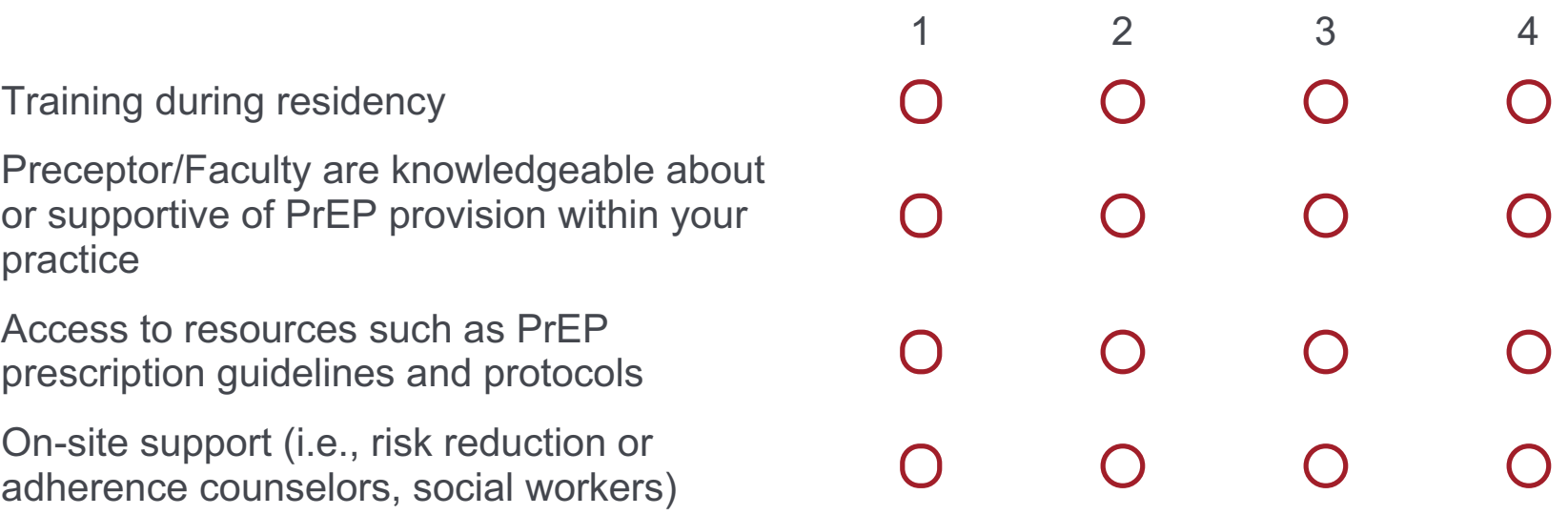

In your opinion, which approach do you think would be most feasible to implement PrEP in your clinical practice setting?

All providers in the practice receive training to provide PrEP and prescribe to eligible patients.

One provider in the practice is appointed as a PrEP specialist (i.e., a provider who receives specific training on PrEP and to whom all eligible patients in the practice are referred)

No providers in the practice receive training or provide PrEP; rather, patients are referred outside the practice (e.g., to an Infectious Disease Clinic or STD clinic)

Other (please specify)

For each of the following risk behavior categories, how comfortable are you evaluating eligibility for PrEP?

$$
\begin{aligned}
& 1=\text { not at all } \\
& \text { comfortable }
\end{aligned}
$$

Women who have sex with men

Men who have sex with women

Men who have sex with men

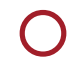

O

O
2

0

0

0
3

0

0

0
4 = very

comfortable

0

\section{(1)}


Men who have sex with men and women

People who inject drugs

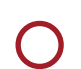

O
O

$\mathrm{O}$
O

$\mathrm{O}$

$\mathrm{O}$
$\mathrm{O}$

For each of the following risk categories, how willing are you to prescribe PrEP to an eligible individual, assuming a recent negative HIV test and equal access to medication:

\begin{tabular}{|c|c|c|}
\hline $\begin{array}{l}1=\text { not at } \\
\text { all willing to } \\
\text { prescribe }\end{array}$ & 2 & 3 \\
\hline
\end{tabular}

A female with a current male partner known to be HIV-positive

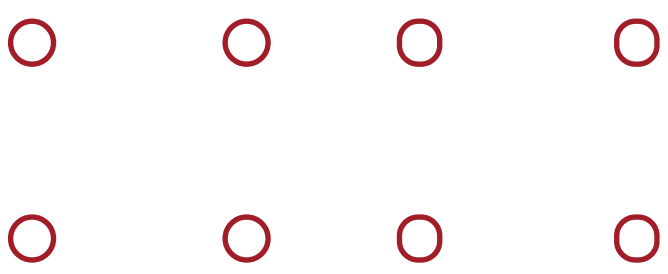

who has sex with other males or uses

injection drugs)

A male with a current female partner known to be HIV-positive

$\mathrm{O}$

O

$\mathrm{O}$

$\mathrm{O}$

A male who has unprotected sex with male partners with unknown HIV status who are at high risk of HIV infection (e.g., partner(s) who has sex with other males or uses injection drugs)

A male with a current male partner known to be HIV-positive

A male who has sex with multiple male partners and has had unprotected anal sex

A person who has a history of multiple prior sexually transmitted illnesses (STIs) (eg chlamydia, gonorrhea)

A person who has injected drugs in the past 6 months and shared injection equipment

A person who has been on methadone maintenance for the past 6 months but has continued injection drug use

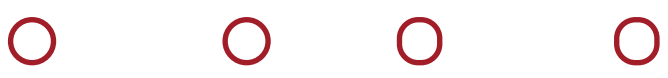

$0 \quad 0 \quad 0$

$0 \quad 0 \quad 0 \quad 0$

O 000

O 000

O $0 \quad 0 \quad 0$

The following questions are about your current practice and career goals: 
What are your future clinical career plans? (Select all that apply)

$$
\begin{gathered}
\text { Primary Care } \\
\text { Specialty Care } \\
\text { HIV Care }
\end{gathered}
$$

I do not plan to practice clinical medicine

Other (please specify)

Do you currently provide direct clinical care in the outpatient setting?

$$
\begin{aligned}
& \text { Yes } \\
& \text { No }
\end{aligned}
$$

In what state is your training program?

How would you classify the geographic area you practice?

Urban

Suburban

Rural

In which settings do you provide care for patients? (Select all that apply including but not limited to your assigned continuity clinic)

Clinic at an academic medical center

Clinic at a public hospital

Clinic at a VA hospital

Clinic at substance abuse treatment center in the community

Community health center

Inpatient/hospital setting 
Private practice (unaffiliated with an academic medical center and in the community) Other (please specify)

What is the main focus of your primary continuity clinic/office where you care for patients? (Select all that apply)

General medical care/Primary Care (but NO HIV care/treatment)

General medical care/Primary Care (WITH HIV care on-site)

Infectious diseases (including HIV care)

Substance abuse treatment

LGBT-focused primary care clinic

Other (please specify)

Of the patients you currently provide care for at your clinic/office, approximately how many patients are HIV-infected?

$$
\begin{gathered}
0 \\
1-10 \\
11-20 \\
21-50 \\
51-100 \\
101+
\end{gathered}
$$

Of the patients you currently provide care for at your office, what percent of those who are eligible for routine HIV testing have been offered testing?

$$
\begin{gathered}
0 \% \\
1-25 \% \\
26-50 \% \\
51-75 \% \\
76-100 \%
\end{gathered}
$$

Not applicable (i.e. all of my patients are HIV+) 
The following questions are demographic questions about you:

What degree have you completed to practice medicine?

DO

MBBS, MBChB, MBBCh, MBBChir, BMBCh, or BMBS

MD

Other (please specify)

What type of residency program are you currently completing?

\author{
Family Medicine \\ Internal Medicine
}

Medicine/Pediatrics (Med/Peds)

Primary Care

Other (please specify)

How long is your training program?

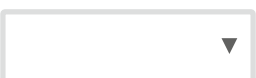

What is your current year of training?

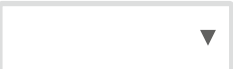

Please enter the year in which you were born? (format YYYY)

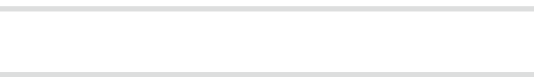

What was your sex assigned at birth (i.e. what was written on your birth certificate at the time of your birth)? 
Female

Male

Prefer not to answer

What is your gender? (Select all that apply)

\author{
Man \\ Woman \\ Genderqueer/Non-conforming \\ Transgender \\ Prefer not to answer
}

With respect to sexual orientation, how do you self-identify?

Heterosexual

Gay

Lesbian

Bisexual

Other (Please specify)

Choose not to answer

With respect to race, how do you self-identify? (Select all that apply)

American Indian and Alaska Native

Asian or Asian American

Black or African American

Native Hawaiian and Pacific Islander

White

Other (please specify)

Prefer not to answer 
Do you self-identify as being Hispanic or Latino?

Yes

No

Prefer not to answer

Thank you for completing the survey.

If you are interested in being entered into a raffle for a $\$ 20$ amazon gift card, please click the following link: where you will be asked to enter your email and contact information. Your information will not be tied to your survey responses.

If you are interested in learning more about PrEP, please consider these resources:

CDC: http://www.cdc.gov/hiv/prevention/research/prep/

Truvada PrEP REMS: http://www.truvadapreprems.com/\#

AIDS Education and Training Center: http://www.aidsetc.org/resource/slideset-prep-hiv-preexposure-prophylaxis 


\section{APPENDIX 2}

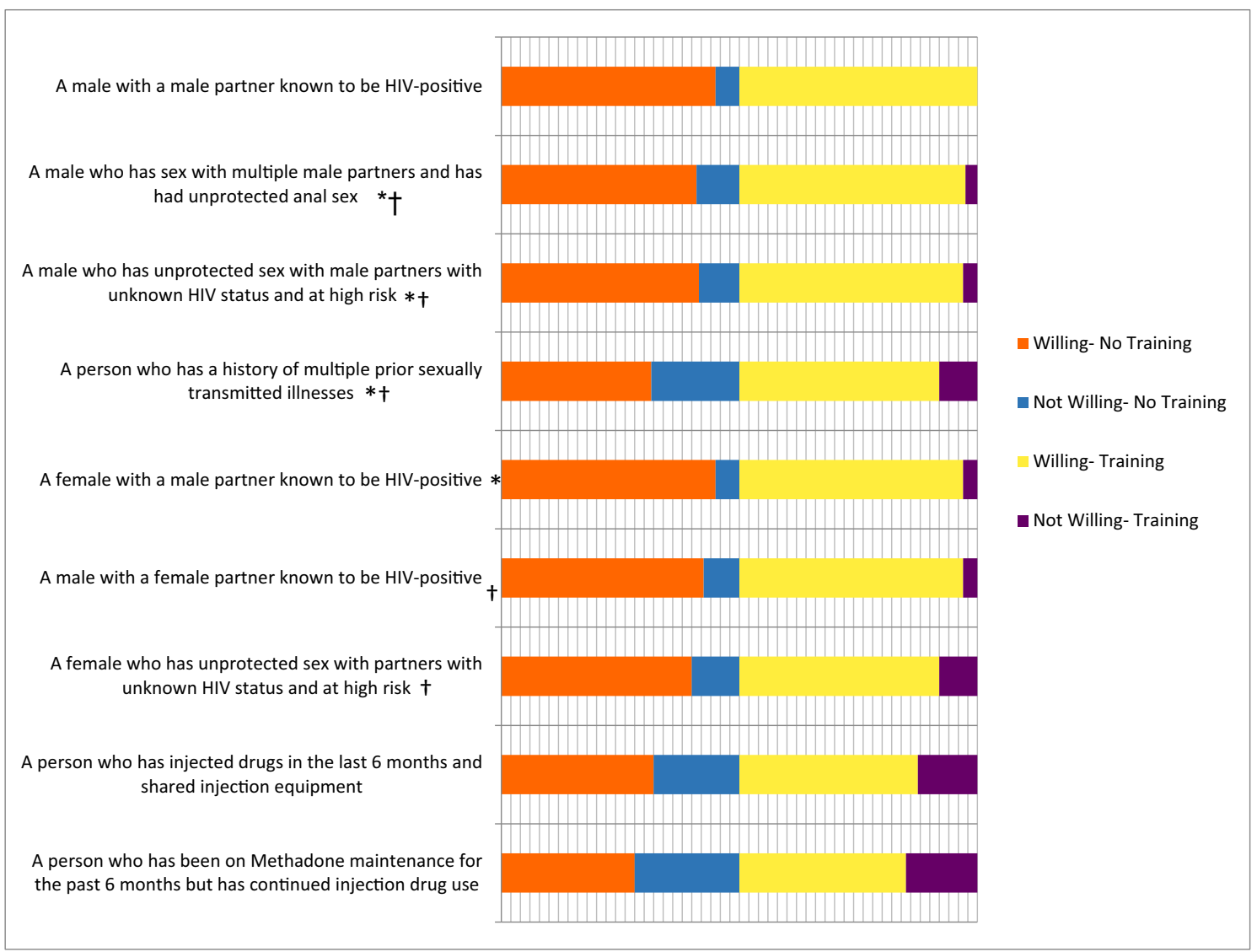

Figure 3 Resident willingness to prescribe PrEP according to HIV risk groups; comparison of residents who report receiving training versus those who did not. $* P<\mathbf{0 . 0 5}$. †required Fisher's exact test for $p$ values due to low counts. 


\section{REFERENCES}

1. Grant RM, Lama JR, Anderson PL, et al. Preexposure chemoprophylaxis for HIV prevention in men who have sex with men. N Engl J Med. 2010; 363:2587-2599.

2. McCormack S, Dunn DT, Desai M, et al. Pre-exposure prophylaxis to prevent the acquisition of HIV-1 infection (PROUD): effectiveness results from the pilot phase of a pragmatic open-label randomized trial. Lancet. 2016; 387(10013):53-60.

3. US Public Health Service. Preexposure Prophylaxis for the Prevention of HIV Infection in the United States- 2017 Update: A Clinical Practice Guideline. Available at: https://www.cdc.gov/hiv/pdf/risk/prep/cdchiv-prep-guidelines-2017.pdf. Accessed December 27, 2018.

4. Center for Disease Control and Prevention. HIV Basics: PrEP. Available at: https://www.cdc.gov/hiv/risk/prep/index.html. Accessed December 27, 2018

5. Tang EC, Sobieszczyk ME, Shu E, Gonzales P, Sanchez J, Lama JR. Provider attitudes toward oral preexposure prophylaxis for HIV prevention among high-risk men who have sex with men in Lima, Peru. AIDS Res Hum Retrovir. 2014; 30(5):416-24.

6. Wilton J, Senn H, Sharma M, Tan DH. Pre-exposure prophylaxis for sexually-acquired HIV risk management: a review. HIV AIDS (Auckl). 2015; 7:125-36.

7. Blumenthal J, Jain S, Krakower D, et al. Knowledge is power! Increased provider knowledge scores regarding pre-exposure prophylaxis (PrEP) are associated with higher rates of PrEP prescription and future intent to prescribe PrEP. AIDS Behav. 2015; 19(5):802-10.

8. Mimiaga MJ, White JM, Krakower DS, Biello KB, Mayer KH. Suboptimal awareness and comprehension of published preexposure prophylaxis efficacy results among physicians in Massachusetts. AIDS Care. 2014; 26(6):684-93.

9. Arnold EA, Hazelton P, Lane T, et al. A qualitative study of provider thoughts on implementing pre-exposure prophylaxis (PrEP) in clinical settings to prevent HIV infection. PLoS One. 2012; 7(7):e40603.

10. Sharma M, Wilton J, Seen H, Fowler S, Tan DH. Preparing for PrEP: perceptions and readiness of Canadian physicians for the implementation of HIV pre-exposure prophylaxis. PLoS One 2014; 9(8):e105283.

11. Blackstock OJ, Moore BA, Berkenblit GV, et al. A cross-sectional online survey of HIV pre-exposure prophylaxis adoption among primary care physicians. J Gen Intern Med. 2017; 32(1):62-70.

12. Eaton LA, Driffin DD, Bauermeister J, Smith H, Conway-Washington C. Minimal awareness and stalled update of pre-exposure prophylaxis (PrEP) among at risk, HIV-negative, black men who have sex with men. AIDS Patient Care STDs. 2015; 29(8):423-29.

13. Barash EA, Golden M. Awareness and use of HIV pre-exposure prophylaxis among attendees of a seattle gay pride event and sexually transmitted disease clinic. AIDS Patient Care STDs. 2010; 21(11):689-91.

14. Mehta SA, Silvera R, Bernstein K, Holzman RS, Aberg JA, Daskalakis DC. Awareness of post-exposure prophylaxis in high-risk men who have sex with men in New York City. Sex Transm Infect. 2011; 87(4):344-48.

15. Smith WR. Evidence for the effectiveness of techniques to change physician behavior. Chest. 2000; 118(2 Suppl):8S-17S.

16. Grohl R, Wensing $\mathbf{M}$. What drives change? Barriers to and incentives for achieving evidence-based practice. Med J Aust. 2004: 180 (6 Suppl):S57-60.

17. Sanazaro P. Continuing education, performance assessment, and quality of patient care. Mobius. 1982; 2:34-37.

18. Berkenblit GV, Sosman JM, Bass M, et al. Factors affecting clinician educator encouragement of routine HIV testing among trainees. J Gen Intern Med. 2012; 27(7):839-44.
19. Mincey BA, Parkulo MA. Antibiotic prescribing practices in a teaching clinic: Comparison of resident and staff physicians. South Med J. 2001; 94:365-9.

20. Ryskina KL, Dine CJ, Kim EJ, Bishop ET, Epstein AJ. Effect of attending practice style on general medication prescribing by residents in the clinic setting: an observational study. J Gen Intern Med. 2015; 30(9): 1286-93.

21. Deville C, Hwang WT, Burgos R, Chapman CH, Both S, Thomas CR Jr. Diversity in graduate medical education in the united states by race, ethnicity, and sex, 2012. JAMA Intern Med. 2015; 175(10):1706-08.

22. Petroll AE, Walsh JL, Owczarzak JL, McAuliffe TL, Bogart LM, Kelly JA. PrEP awareness, familiarity, comfort, and prescribing experience among us primary care providers and HIV specialists. AIDS Behav. 2017;21(5): 1256-1267.

23. White JM, Mimiaga MJ, Krakower DS, Mayer KH. Evolution of Massachusetts physician attitudes, knowledge, and experience regarding the use of antiretrovirals for HIV prevention. AIDS Patient Care STDS. 2012; 26(7):395-405

24. Prior M, Guerin M, Grimmer-Somers K. The effectiveness of clinical guideline implementation strategies - a synthesis of systematic review findings. J Eval Clin Pract. 2008; 14(5):888-97.

25. Choudhry NK, Fletcher RH, Soumerai SB. Systematic review: the relationship between clinical experience and quality of health care. Ann Intern Med. 2005; 142(4):260-73.

26. Krakower D, Mayer KH. Engaging healthcare providers to implement HIV pre-exposure prophylaxis. Curr Opin HIV AIDS. 2012; 7(6):593-99.

27. Silapaswan A, Krakower D, Mayer KH. Pre-exposure prophylaxis: a narrative review of provider behavior and interventions to increase prep implementation in primary care. J Gen Intern Med. 2017; 32(2):192-198.

28. Edelman EJ, Moore BA, Calabrese SK, et al. Primary care physician's willingness to prescribe hiv pre-exposure prophylaxis for people who inject drugs. AIDS Behav. 2017; 21(4):1025-33.

29. Krakower D, Ware N, Mitty JA, Maloney K, Mayer KH. HIV providers' perceived barriers and facilitators to implementing pre-exposure prophylaxis in care settings: a qualitative study. AIDS Behav. 2014; 18(9):171221 .

30. Marcus JL, Volk JE, Pinder J, et al. Successful implementation of HIV preexposure prophylaxis: lessons learned from three clinical settings. Curr HIV/AIDS Rep. 2016; 13(2):116-24.

31. Goldstein RH, Streed CG Jr, Cahill SR. Being PrEPared - preexposure prophylaxis and HIV disparities. N Engl J Med. 2018; 379(14):12931295.

32. Scherer ML, Douglas NC, Churnet BH, et al. Survey of HIV care providers on management of HIV serodiscordant couples- assessment of attitudes, knowledge and practices. AIDS Care. 2014; 26(11):1435-39.

33. McMahon JM, Myers JE, Kurth AE, et al. Oral pre-exposure prophylaxis (PrEP) for prevention of HIV in serodiscordant heterosexual couples in the United States: opportunities and challenges. AIDS Patient Care STDS. 2014; 28(9):462-74

34. Hong JN, Farel CE, Rahangdale L. Pharmacologic prevention of human immunodeficiency virus in women: practical approaches for the obstetrician and gynecologist. Obstet Gynecol Surv. 2015; 70(4):284-90.

35. Kaplowitz MD, Hadlock TD, Levine R. A comparison of web and mail survey response rates. Public Opin Q. 2004; 68 (1): 94-101.

36. Grava-Gubins I, Scott S. Effects of various methodologic strategies: survey response rates among Canadian physicians and physicians-in training. Can Fam Physician. 2008; 54(10): 1424-30.

Publisher's Note Springer Nature remains neutral with regard to jurisdictional claims in published maps and institutional affiliations. 